\title{
Efficient combination of feedforward and feedback structures for nonlinear narrowband active noise control
}

\author{
Luo Lei, Jinwei Sun *, Boyan Huang, Dung Duong Quoc \\ School of Electrical Engineering \& Automation, Harbin Institute of Technology, Harbin 150001, China
}

\begin{abstract}
In nonlinear narrowband active noise control (NNANC) system, there often exist nonlinear distortions in such cases: the primary path is nonlinear and the secondary path is non-minimum phase. In order to solve these nonlinear issues, a novel NNANC scheme based the combination of feedforward and feedback structures is proposed in this paper. In new NNANC scheme, the spectrum information fed back from the noise cancelling point is corrected by the frequency components expanded from the reference signal to obtain precise frequency components of primary noise. Then these frequency components are used as the input signal of new NNANC scheme and each frequency component is processed independently to reduce the undesired noise. For reducing the computational complexity and simplifying the implementation structure of new NNANC scheme, the filtered-error structure is used in proposed scheme to form Proposed-1 algorithm. To improve the performance of Proposed-1 algorithm even further, error separation technique using bandpass filter banks for these frequency components is developed to form Proposed-2 algorithm. Computational complexity analysis and numerous simulations for different narrowband noise and nonlinear primary path are given to demonstrate the effectiveness of proposed schemes.
\end{abstract}

Keywords: nonlinear active noise control, feedforward and feedback structures, filtered-error structure, error separation

\section{Introduction}

Nowadays, excessive noise has a serious impact on people's health, especially the older and the infant. In some extent, the noise has become an invisible killer. With much more attention paid to this problem, various methods have been proposed over the past few decades. Originally, passive noise control (PNC) system using passive absorber is applied to reduce the noise. This method performs well on the high frequency noise but poorly even invalidly on the low frequency noise. Compared with PNC system, the active noise control (ANC) system put forward in the 1970s has advantages to suppressing low frequency acoustic noise with much smaller size and lower cost $[1,2]$.

The ANC system is an electro-acoustic device based on the principle of destructive interference by generating an anti-noise with same amplitude, frequency and opposite phase of the undesired noise. Because of potential industrial applications and advances in digital signal processing, ANC has become one of the key research areas in recent years. ANC system can be

\footnotetext{
* Corresponding author. E-mail addresses: jwsun@ @it.edu.cn
} 
classified broadband ANC (BANC) system and narrowband ANC (NANC) system according to frequency characteristic [3]. Compared with BANC system, the NANC system is employed more widely in controlling industrial noise. Narrowband noise is usually produced by rotating machines, such as fans, diesel engines, motors, cutting machines, etc. these noise signals may be modelled as the combination of different sinusoidal signals. The conventional NANC system is found to be effective in suppressing undesirable sinusoidal noise in many real-life applications [4]. The discrete Fourier coefficients (DFCs) of sine and cosine are adjusted by filtered-x least mean square (FXLMS) algorithm which has been widely used because of its simpler structure and lower computational load [4-6]. However, these superiorities are limited to linear control problem, in other words, the reference signal measured by acoustic sensor or non-acoustic sensor and the acoustic paths must be linear entirely. As a consequence, the FXLMS algorithm based on NANC system performs poorly when the primary path is nonlinear and the secondary path is non-minimum phase [7-12].

To solve the problems of NNANC, a variety of sophisticated and efficient nonlinear control structures have been raised, such as the functional link artificial neural network (FLANN) using filtered-s least mean square (FSLMS) algorithm [13-18] and the Volterra FXLMS (VFXLMS) algorithm using adaptive Volterra filter [19-21], these two methods are the most potential nonlinear adaptive feedforward structures in the nonlinear ANC field. Compared with the VFXLMS algorithm, the FSLMS algorithm is paid more attention, because the FSLMS algorithm has more uncomplicated computation load in most cases, but when the length of secondary path estimate is shorter, the VFXLMS algorithm performs more excellently. Recently, many other algorithms have been proposed to improve the performance of nonlinear ANC system. The faster and exacter versions of the FSLMS and VFXLMS algorithms are introduced in paper [22] to decrease computational load. Adaptive reduced feedback functional link neural network (RFFLNN) filter for nonlinear ANC system with better convergence speed and steady-state error is proposed in paper [23]. However, in NNANC system, the target signal processed by these algorithms is the whole input signal not one certain frequency wave, which results in some unbalance in the noise cancelling procedure and limits them to be improved further on convergence rate and steady-state error. Even though the expansion order of these schemes is enlarged, which leads to ever increasing number of computational burden, the result performs still poorly. By analysing the NNANC system composed of nonlinear primary path and non-minimum phase secondary path, what can be found is that the primary noise is narrowband and its frequency components are predictable. Therefore, we proposed new NNANC schemes composed of feedforward and feedback structures with lower computational load in this paper. The result of frequency expansion of reference signal is used to correct feedback output wave to obtain the final input signal of the proposed NNANC schemes. By this way, the frequency components of input signal are the same as that of primary noise.

The organization of this paper is as follows. In Section 2, the NANC system is introduced and its primary noise is analysed in the case of linear and nonlinear primary path respectively. On the basis of analysing primary noise of NNANC system, a new 
NNANC scheme composed of feedforward and feedback structures with filtered-error algorithm (Proposed-1) and its improved version using error separation technique (Proposed-2) are developed in section 3. The comparison of the computational load of feedforward structure like FSLMS algorithm and VFXLMS algorithm, the combination of feedforward structure and feedback structure like RFFLNN algorithm and proposed schemes is also presented in this section. Section 4 provides computer simulation studies of the proposed schemes. Finally, conclusion is presented in Section 5.

\section{Narrowband Active Noise Control}

In narrowband active noise control (NANC) system, the frequencies of reference signal may be calculated from a synchronization (Sync) signal acquired by a timing signal sensor such as tachometer, so the reference signal is given by

$$
x(n)=\sum_{i=1}^{q}\left[a_{i} \cos \left(\omega_{i}(n)\right)+b_{i} \sin \left(\omega_{i}(n)\right)\right]
$$

where $\left(\omega_{q} \geq \omega_{q-1} \geq \cdots \geq \omega_{2} \geq \omega_{1}\right), q$ is the number of frequency components in reference signal, $\left\{a_{i}, b_{i}\right\}_{i=1}^{q}$ are the discrete Fourier coefficients (DFCs) of frequency components $\left\{\omega_{i}\right\}_{i=1}^{q}$.

\subsection{Linear Primary Path}

The conventional linear NANC (LNANC) system is depicted in Fig.1, whose controller is linear [24], where $v_{p}(n)$ is additive white Gaussian noise which acts as background noise. This system has been found very effective and efficient to suppress primary sinusoidal noise signal generated by rotating machines. When the transfer function of primary path is linear, compared with reference signal, there is no new frequency components are produced in primary noise. As a result, the primary noise is expressed by

$$
p(n)=\sum_{i=1}^{q}\left[a_{p, i} \cos \left(\omega_{i} n\right)+b_{p, i} \sin \left(\omega_{i} n\right)\right]
$$

where $\left\{a_{p, i}, b_{p, i}\right\}_{i=1}^{q}$ are the DFCs of primary noise.

Owing to the frequency components are unchanged in the LNANC, the secondary source signal is generated directly by control filters placed in parallel form. These control filters are combined linearly, each of them takes care of one target frequency. The sum of output signal of all control filters forms the secondary source signal, which can be expressed by

$$
\begin{aligned}
y(n) & =\sum_{i=1}^{q} y_{i}(n) \\
& =\sum_{i=1}^{q}\left[\hat{a}_{i}(n) \cos \left(\omega_{i} n\right)+\hat{b}_{i}(n) \sin \left(\omega_{i} n\right)\right]
\end{aligned}
$$

where $\left\{\hat{a}_{i}(n), \hat{b}_{i}(n)\right\}_{i=1}^{q}$ are the DFCs of secondary noise, which are usually updated online by FXLMS algorithm or filtered-X 
recursive least squares (FXRLS) algorithm.

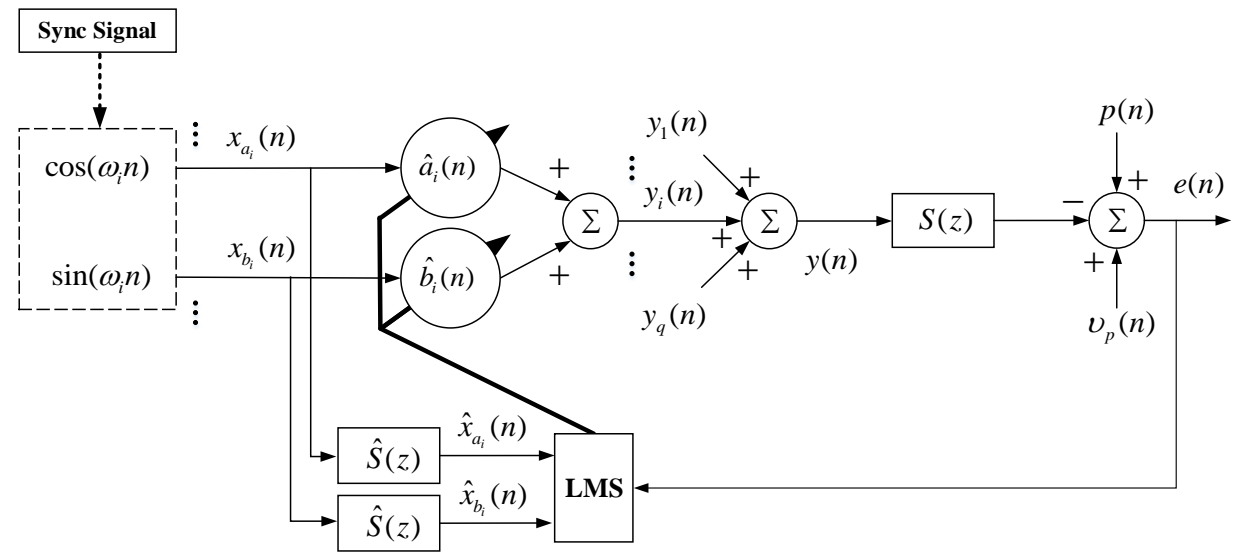

Fig. 1 The block diagram of conventional LNANC

\subsection{Nonlinear Primary Path}

If the transfer function of primary path is nonlinear, the frequency components of primary noise may be changed and different from the frequency components of reference signal, this problem will result in the conventional LNANC structure works inefficiently and even worse.

Generally, in the ANC system, the primary path is always the continuous function which can be expressed by polynomial using the theory of Taylor expansion, since the primary noise can be given by

$$
\begin{aligned}
p(n) & =\sum_{t=1}^{T}\left(c_{t, 0} x(n)+c_{t, 1} x(n-1)+\cdots+c_{t, m} x(n-m)\right)^{t} \\
& =\sum_{t=1}^{T}\left[\sum_{t_{1}=0}^{t} \sum_{t_{2}=0}^{t_{1}} \cdots \sum_{t_{m}=0}^{t_{m-1}}\left(\frac{t !}{t_{m} !\left(t-t_{1}\right) ! \cdots\left(t_{m-1}-t_{m}\right) !}\right)\left(c_{t, 0} x(n)\right)^{t-t_{1}}\left(c_{t, 1} x(n-1)\right)^{t_{1}-t_{2}} \cdots\left(c_{t, m} x(n-m)\right)^{t_{m}}\right]
\end{aligned}
$$

where $T \in \mathbf{Z}_{+}$is the order of the primary path, when it is equal to one, the primary path is linear, otherwise, the primary path is nonlinear.

Substituting the Eq. (1) into the function $(\bullet)^{k}$

$$
\begin{aligned}
(x(n))^{k} & =\left\{\sum_{i=1}^{q}\left[a_{i} \cos \left(\omega_{i} n\right)+b_{i} \sin \left(\omega_{i} n\right)\right]\right\}^{k} \\
& =\sum_{i=1}^{I}\left(a_{i}^{\prime} \cos \left(\left(h_{i, 1} \omega_{1}+h_{i, 2} \omega_{2}+\cdots+h_{i, q} \omega_{q}\right) n\right)+b_{i}^{\prime} \sin \left(\left(h_{i, 1} \omega_{1}+h_{i, 2} \omega_{2}+\cdots+h_{i, q} \omega_{q}\right) n\right)\right)
\end{aligned}
$$

where $I=\left\{0<\left|h_{i, 1}\right|+\left|h_{i, 2}\right|+\cdots+\left|h_{i, q}\right| \leq k \mid h_{i, 1}, h_{i, 2}, \cdots h_{i, q} \in \mathbf{Z}\right\}$. The Eq. (5) is substituted into the Eq. (4) to get primary noise

$$
p(n)=\sum_{i=1}^{Q}\left(a_{p, i} \cos \left(\left(d_{i, 1} \omega_{1}+d_{i, 2} \omega_{2}+\cdots+d_{i, q} \omega_{q}\right) n\right)+b_{p, i} \sin \left(\left(d_{i, 1} \omega_{1}+d_{i, 2} \omega_{2}+\cdots+d_{i, q} \omega_{q}\right) n\right)\right)
$$

where $Q=\left\{0<\left|d_{i, 1}\right|+\left|d_{i, 2}\right|+\cdots+\left|d_{i, q}\right| \leq T \mid d_{i, 1}, d_{i, 2}, \cdots d_{i, q} \in \mathbf{Z}\right\}$. 
The frequency scope of primary noise can be expressed by

$$
\min \left\{\left|d_{i, 1} \omega_{1}+d_{i, 2} \omega_{2}+\cdots+d_{i, q} \omega_{q}\right|\right\} \leq \omega \leq T \omega_{q}
$$

Analysis above, when the frequencies of reference signal and the order of primary path are known, the frequencies of primary noise will be determined. However, the order of primary path is usually unknown in practical application. Considering the sampling frequency $f_{s}$, the frequency scope of primary noise is rewritten as

$$
\min \left\{\left|d_{i, 1} \omega_{1}+d_{i, 2} \omega_{2}+\cdots+d_{i, q} \omega_{q}\right|\right\} \leq \omega \leq 0.5 f_{s}
$$

\section{The Proposed NNANC Schemes}

It has been introduced above that the primary noise of NNANC system is also narrowband signal when the reference signal is narrowband, so a new NNANC scheme is proposed in this paper, block diagram of new NNANC scheme is depicted in Fig.2. In this scheme, the narrowband frequency components of primary noise are used as the input signal to control undesired noise, because the control targets are more accurate, the performance on noise cancellation will be better. The residual noise measured by the error sensor is presented as

$$
e(n)=p(n)-y(n) * s(n)+v_{p}(n)
$$

where $s(n)$ is the impulse response of the transfer function $S(z)$ and * is the convolution operator, $v_{p}(n)$ is additive background noise.

In the fact, the primary noise $p(n)$ can't be obtained directly, so its estimate value $g(n)$ is regarded as substitution. $g(n)$ is given by

$$
g(n)=e(n)+y(n) * \hat{s}(n)
$$

where $\hat{S}(n)$ is the impulse response of the transfer function $\hat{S}(z)$, and $\hat{S}(z)$ is the estimate of $S(z)$. Compared with $p(n)$, the $v_{p}(n)$ is smaller. Therefore, when $\hat{S}(z)$ is estimated accurately, the estimate value $g(n)$ can replace the primary noise $p(n)$ to obtain the frequency components of noise canceling point.

\subsection{Feedback Structure}

In Fig. 2, the feedback structure is used to estimate the frequency information of primary noise. Many techniques have been used to estimate the signal frequency such as discrete Fourier transform, least-squares technique, Kalman filtering, recursive Newton-type algorithm, adaptive notch filters, multiple signal classification (MUSIC), minimum variance distortionless response (MVDR) spectrum. Though these methods can acquire more accurate frequency, they suffers from heavier computational burden and is used difficultly in practical applications, especially the ANC system which focuses on the teal-time signal processing 
greatly. Furthermore, the frequencies estimated by feedback structure is not concerned on accuracy, because these frequencies will be corrected by the frequencies expanded from reference signal in feedforward structure.

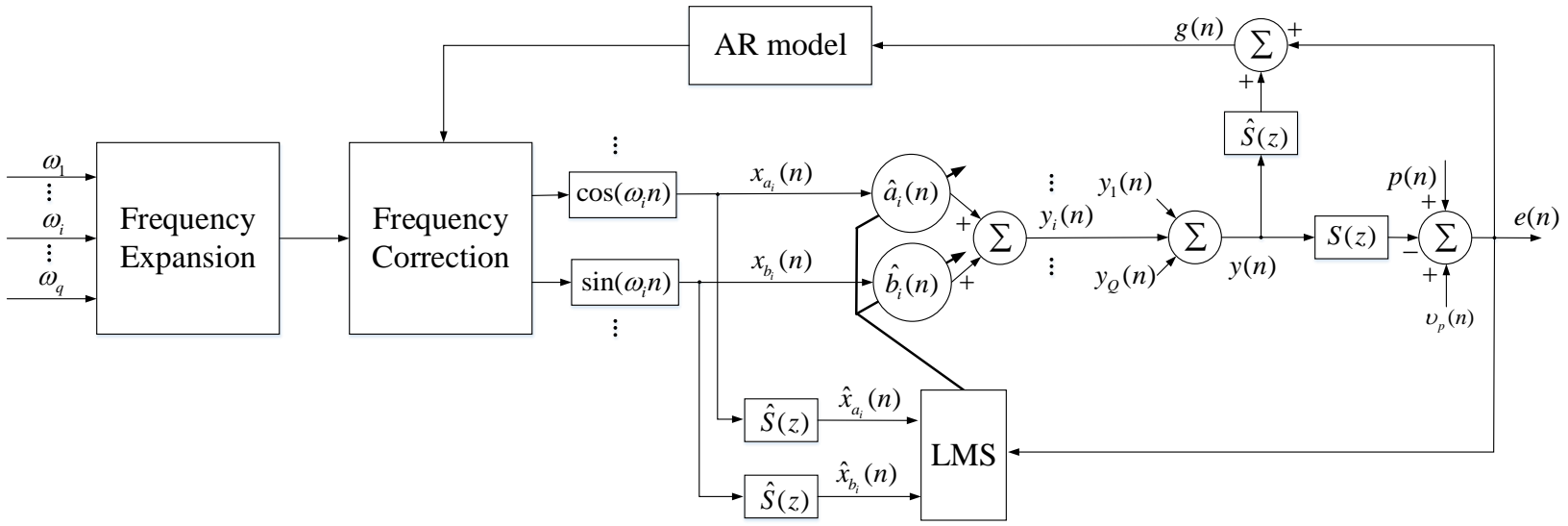

Fig. 2 Schematic diagram of new NNANC scheme composed of feedforward and feedback structures

In view of the analysis above, the autoregressive (AR) model is used to estimate the spectrum of primary noise in this paper. If the order of AR model is long enough, better performance can also be obtained. An AR model refers to a system in which the output signal depends linearly on its previous value, so the estimate value of primary noise can be expressed by

$$
g(n)=\sum_{i=1}^{L} a_{R, i} g(n-i)+v(n)
$$

where $a_{R, i}$ are the coefficients of AR model and $L$ is its order, $v(n)$ is an error term. If the $v(n)$ is regarded as the stimulated signal, the transfer function of an AR model is only composed of poles

$$
H(z)=\frac{1}{1-\sum_{i=1}^{L} a_{R, i} z^{-i}}
$$

When the $L$ is long enough, the spectrum of $g(n)$ is given by

$$
P_{g}\left(e^{j w}\right)=\frac{\sigma_{v}{ }^{2}}{\left|1-\sum_{i=1}^{L} a_{R, i} e^{-j w i}\right|^{2}}
$$

where $\sigma_{v}{ }^{2}$ is the variance of $v(n)$, because the value and the number of frequencies are only focused on this paper, the molecule of $P_{g}\left(e^{j w}\right)$ is equal to one constantly in calculation.

Linear prediction (LP) is an efficient and sophisticated way to get the coefficients of an AR mode which is always used to analyse the signal's spectrum. If the spectrum of the stimulus signal is supposed to be flat, the LP coefficients can be regarded as the AR model coefficients. The LP coefficients can be calculated online by adaptive AR model which is shown in Fig. 3. 
$\left\{a_{L P, i}(n)\right\}_{i=1}^{L}$ are the LP coefficients at time index $n$, which can be updated by LMS algorithm as follow

$$
\begin{gathered}
v(n)=g(n)-\sum_{i=1}^{L} a_{L P, i}(n) g(n-i) \\
a_{L P, i}(n+1)=a_{L P, i}(n)+\mu_{g} v(n) g(n-i)
\end{gathered}
$$

where $\mu_{g}$ is the step size for updating.

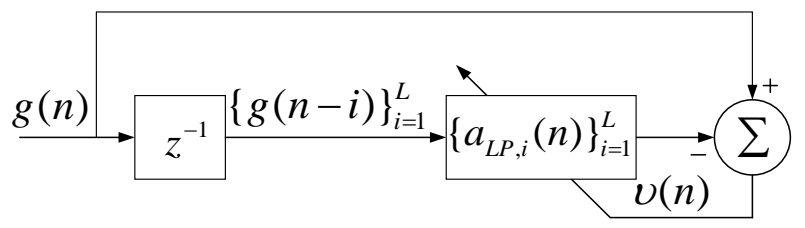

Fig .3 Adaptive AR model

Through the AR model based on LMS algorithm, the spectrum of primary noise is obtained roughly. Because the value and the number of frequencies are needed, the maximum of the spectrum must be known. Generally, the maximum can be searched directly from $f=0$ to $f=0.5 f_{s}$ one by one. This research requires $0.5 f_{s}$ times calculation to obtain all maximum, straightforward but costly.

\subsection{Feedforward Structure}

By analysing the frequency property of primary noise of NNANC system, the frequency scope of primary noise can be calculated by the frequencies of reference signal and the sampling frequency. Therefore, we expand the frequencies of reference signal within half of the sampling frequency like Eq. (8) using feedforward structure. By this way, the possible frequency components of primary noise can be determined initially. Then the estimated range may be divided into small bins around these expanded frequencies and every bin can be seen one-dimensional optimization problem. The Golden Section Search (GSS) is the best candidate to seek the maximum of the spectrum, because it can reduce the computational cost dramatically.

The GSS is the technique to find extremum (minimum or maximum) by narrowing the estimation range continuously at a constant ratio which is commonly known as the golden ratio in mathematics. In this paper, the maximum error of $i$ th estimated frequency is $\tau_{i}$, so each bin can be expressed as $\left[-\tau_{i}+\omega_{e, i}, \tau_{i}+\omega_{e, i}\right]$. The $\omega_{e, i}$ is $i$ th expanded frequency

$$
\omega_{e, i}=\left\{\left|d_{i, 1} \omega_{1}+d_{i, 2} \omega_{2}+\cdots+d_{i, q} \omega_{q}\right| \leq 0.5 f_{s}\right\}
$$

the number of expanded frequencies is $Q^{\prime}$, usually $Q^{\prime} \geq Q$.

Two points $p_{i, 1}$ and $p_{i, 2}$ are selected in each internal $\left(-\tau_{i}+\omega_{e, i}, \tau_{i}+\omega_{e, i}\right)$ to divide this internal into three parts. $p_{i, 1}$ and $p_{i, 2}$ are delimited as 


$$
\begin{aligned}
& p_{i, 1}=\lambda\left(\omega_{e, i}-\tau_{i}\right)+(1-\lambda)\left(\omega_{e, i}+\tau_{i}\right) \\
& p_{i, 2}=(1-\lambda)\left(\omega_{e, i}-\tau_{i}\right)+\lambda\left(\omega_{e, i}+\tau_{i}\right)
\end{aligned}
$$

where $\lambda$ is the golden radio. Comparing the amplitude of these two points, if $f\left(p_{i, 1}\right)<f\left(p_{i, 2}\right)$, the maximum lies on the internal $\left[p_{i, 1}, \tau_{i}+\omega_{e, i}\right]$, otherwise, the maximum lies on the internal $\left[-\tau_{i}+\omega_{e, i}, p_{i, 2}\right]$. By this way, the effective rang will be narrowed. Similarly, another two points are inserted in the reduced interval to form a new search interval for next iteration. After the repeated search for a certain number or when the search interval is shrunk to a desired tolerance, the search will be stopped to get the maximum point. If the maximum point is $-\tau_{i}+\omega_{e, i}$ or $\tau_{i}+\omega_{e, i}$, this frequency will be rejected.

When the frequency components $\left\{\omega_{i}^{\prime}\right\}_{i=1}^{Q}$ of primary noise are acquired, the secondary noise can be expressed by

$$
\begin{aligned}
y(n) & =\sum_{i=1}^{Q} y_{i}(n) \\
& =\sum_{i=1}^{Q}\left[\hat{a}_{i}(n) \cos \left(\omega_{i}^{\prime} n\right)+\hat{b}_{i}(n) \sin \left(\omega_{i}^{\prime} n\right)\right]
\end{aligned}
$$

where $\left\{\hat{a}_{i}(n), \hat{b}_{i}(n)\right\}_{i=1}^{Q}$ are the DFCs of all frequency components of secondary noise. When the real secondary path is nonminimum phase, the residual noise $e(n)$ can be expressed by

$$
\begin{aligned}
e(n) & =p(n)-\sum_{m=0}^{M} s_{m} y(n-m)+v_{p}(n) \\
& =\sum_{i=1}^{Q}\left\{\left[\left(a_{p, i}-a_{y, i}(n)\right) \cos \left(\omega_{i}^{\prime} n\right)+\left(b_{p, i}-b_{y, i}(n)\right) \sin \left(\omega_{i}^{\prime} n\right)\right]\right\}+v_{p}(n)
\end{aligned}
$$

where $\left\{a_{y, i}(n), b_{y, i}(n)\right\}_{i=1}^{Q}$ are the output of $\left\{\hat{a}_{i}(n), \hat{b}_{i}(n)\right\}_{i=1}^{Q}$ filtered by secondary path $S(z)$ whose order is $M$. When $\left\{a_{y, i}(n), b_{y, i}(n)\right\}_{i=1}^{Q}$ are equal to $\left\{a_{p, i}, b_{p, i}\right\}_{i=1}^{Q}$ respectively, the residual noise $e(n)$ reaches its desired value $v_{p}(n)$.

\subsection{New NNANC scheme with filtered-error structure (Proposed-1)}

In Fig. 2, the sinusoidal and cosine components of each frequency must be filtered by estimated secondary path. Therefore, the number of $\mathrm{x}$-filtering blocks will increase quickly as the number of target frequencies become larger, which leads to a serious cost issue in practical application. In paper [25-27], the nonlinear ANC system with filtered-error structure makes a great contribution on this problem, where only the error signal needs to be filtered by an error filter rather than filtering every component of input signal, so great savings in the computation and memory requirements can be achieved. Referring to this scheme, a new NNANC system with filtered-error structure called Proposed-1 in this paper is shown in Fig. 4. 


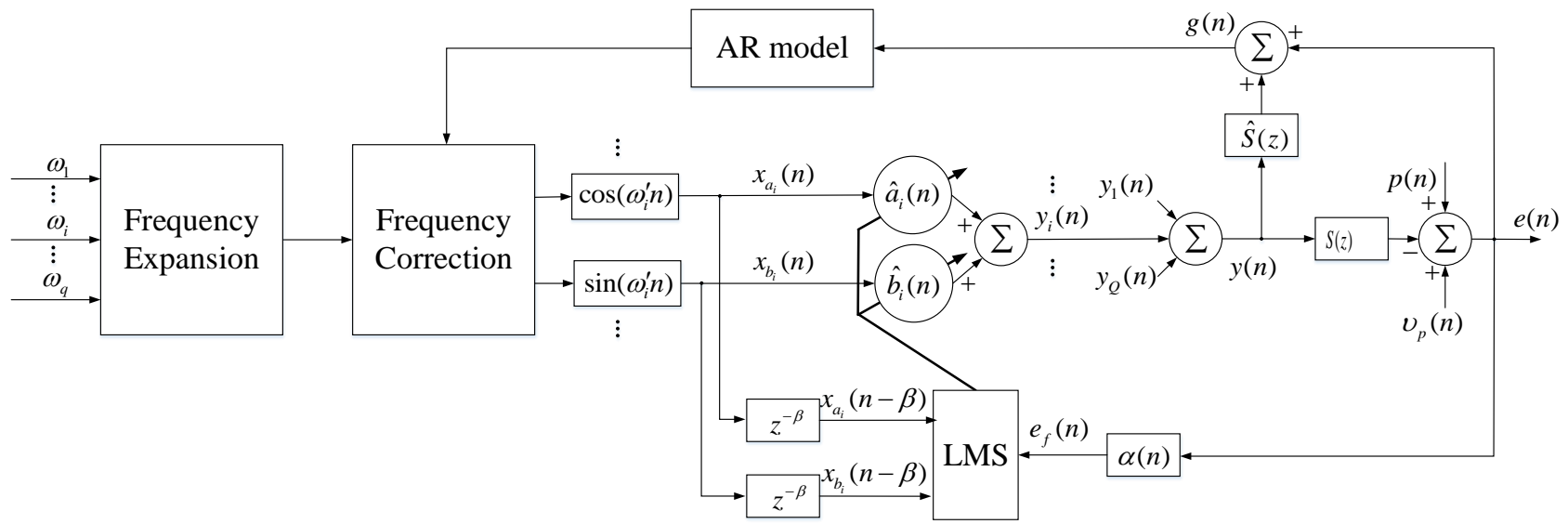

Fig. 4 Schematic diagram of proposed NNANC scheme with filtered-error structure (Proposed-1)

In Proposed-1, the DFCs $\left\{\hat{a}_{i}(n), \hat{b}_{i}(n)\right\}_{i=1}^{Q}$ are updated by FXLMS algorithm

$$
\begin{aligned}
& \hat{a}_{i}(n+1)=\hat{a}_{i}(n)+\mu_{a} \Delta \hat{a}_{i} \\
& \hat{b}_{i}(n+1)=\hat{b}_{i}(n)+\mu_{b} \Delta \hat{b}_{i}
\end{aligned}
$$

where

$$
\begin{aligned}
& \Delta \hat{a}_{i}=e(n) \sum_{m=0}^{\hat{M}} \hat{s}_{m} \cos \left(\omega_{i}^{\prime}(n-m)\right) \\
& \Delta \hat{b}_{i}=e(n) \sum_{m=0}^{\hat{M}} \hat{s}_{m} \sin \left(\omega_{i}^{\prime}(n-m)\right)
\end{aligned}
$$

where parameter $\hat{M}$ and $\left\{\hat{s}_{m}\right\}_{m=0}^{\hat{M}}$ are the order and coefficients of the secondary path estimate $\hat{S}(z)$. Usually $\hat{S}(z)$ is determined offline in advance or online by some parameter identification technique such as the LMS algorithm, Wiener filter etc..

Let $k=n-m+\hat{M}$, so that $n=k+m-\hat{M}$

$$
\begin{gathered}
\Delta \hat{a}_{i}=\sum_{m=0}^{\hat{M}} e(k+m-\hat{M}) \hat{s}_{m} \cos \left(\omega_{i}^{\prime}(k-\hat{M})\right) \\
\Delta \hat{b}_{i}=\sum_{m=0}^{\hat{M}} e(k+m-\hat{M}) \hat{s}_{m} \sin \left(\omega_{i}^{\prime}(k-\hat{M})\right)
\end{gathered}
$$

let $j=\hat{M}-m$, then

$$
\begin{gathered}
\Delta \hat{a}_{i}=\sum_{j=0}^{\hat{M}} e(n-j) \hat{s}_{\hat{M}-j} \cos \left(\omega_{i}^{\prime}(n-\hat{M})\right) \\
\Delta \hat{b}_{i}=\sum_{j=0}^{\hat{M}} e(n-j) \hat{s}_{\hat{M}-j} \sin \left(\omega_{i}^{\prime}(n-\hat{M})\right)
\end{gathered}
$$


Supposing that

$$
e_{f}(n)=\sum_{j=0}^{\hat{M}} \hat{s}_{\hat{M}-j} e(n-j)
$$

substituting Eq. (27)-(29) into Eq. (21) and (22), and let $\mu_{P}=\mu_{a}=\mu_{b}$ to simplify the updated process.

$$
\begin{aligned}
& \hat{a}_{i}(n+1)=\hat{a}_{i}(n)+\mu_{P} e_{f}(n) \cos \left(\omega_{i}^{\prime}(n-\hat{M})\right) \\
& \hat{b}_{i}(n+1)=\hat{b}_{i}(n)+\mu_{P} e_{f}(n) \sin \left(\omega_{i}^{\prime}(n-\hat{M})\right)
\end{aligned}
$$

According to above equations, we can know that $\beta=\hat{M}$ and $\alpha(n)=\left[\begin{array}{llll}\hat{s}_{\hat{M}} & \hat{s}_{\hat{M}-1} & \cdots & \hat{s}_{0}\end{array}\right]$.

\subsection{Improvement of Proposed-1}

For further improvement, the filtered-error signal of Proposed-1 is separated by bandpass filter banks in this section. The improved structure is called Proposed-2, which is shown in Fig. 5. The transfer function of $i$ th bandpass filter is expressed by

$$
\begin{gathered}
H_{b p, i}(z)=\frac{(\rho-1) r_{i} z^{-1}+\left(\rho^{2}-1\right) z^{-2}}{1+\rho r_{i} z^{-1}+\rho^{2} z^{-2}} \\
r_{i}=-2 \cos \left(\omega_{i}^{\prime}\right)
\end{gathered}
$$

where $\rho$ is a pole attraction factor (or pole radius) over $(0,1)$. The bandwidth of the bandpass filter can be presented as $(1-\rho) \pi$. It is clear that the bandwidth is easily adjusted to an intended value by selecting a proper $\rho$.

In Proposed-1, the error signal $e(n)$ is composed by

$$
e(n)=\sum_{i=1}^{Q} e_{i}(n)+v_{p}(n)
$$

Combining (29) and (34), $e_{f}(n)$ can be rewritten as

$$
\begin{aligned}
e_{f}(n) & =\sum_{i=1}^{Q}\left[\sum_{m=0}^{\hat{M}} \hat{s}_{\hat{M}-m} e_{i}(n-m)\right]+\left[\sum_{m=0}^{\hat{M}} \hat{s}_{\hat{M}-m} v_{p}(n-m)\right] \\
& =\sum_{i=1}^{Q} e_{f, i}(n)+\varsigma(n)
\end{aligned}
$$

where $\varsigma(n) \ll \sum_{i=1}^{Q} e_{f, i}(n) . e_{f, i}(n)$ is caused by $i$ th frequency component and is irrelevant with each other, so the DFCs of each frequency component updated by FXLMS algorithm can be also expressed accurately by

$$
\begin{aligned}
& \hat{a}_{i}(n+1)=\hat{a}_{i}(n)+\mu_{P} \hat{e}_{f, i}(n) \cos \left(\omega_{i}^{\prime}(n-\hat{M})\right) \\
& \hat{b}_{i}(n+1)=\hat{b}_{i}(n)+\mu_{P} \hat{e}_{f, i}(n) \sin \left(\omega_{i}^{\prime}(n-\hat{M})\right)
\end{aligned}
$$


where $\hat{e}_{f, i}(n)$ is extracted from filtered-error $e_{f}(n)$ by a bandpass filter shown in Eq. (32). Therefore, $\hat{e}_{f, i}(n)$ is given by

$$
\hat{e}_{f, i}(n)=-\rho r_{i} \hat{e}_{f, i}(n-1)-\rho^{2} \hat{e}_{f, i}(n-2)+(\rho-1) r_{i} e_{f}(n-1)+\left(\rho^{2}-1\right) e_{f}(n-2)
$$

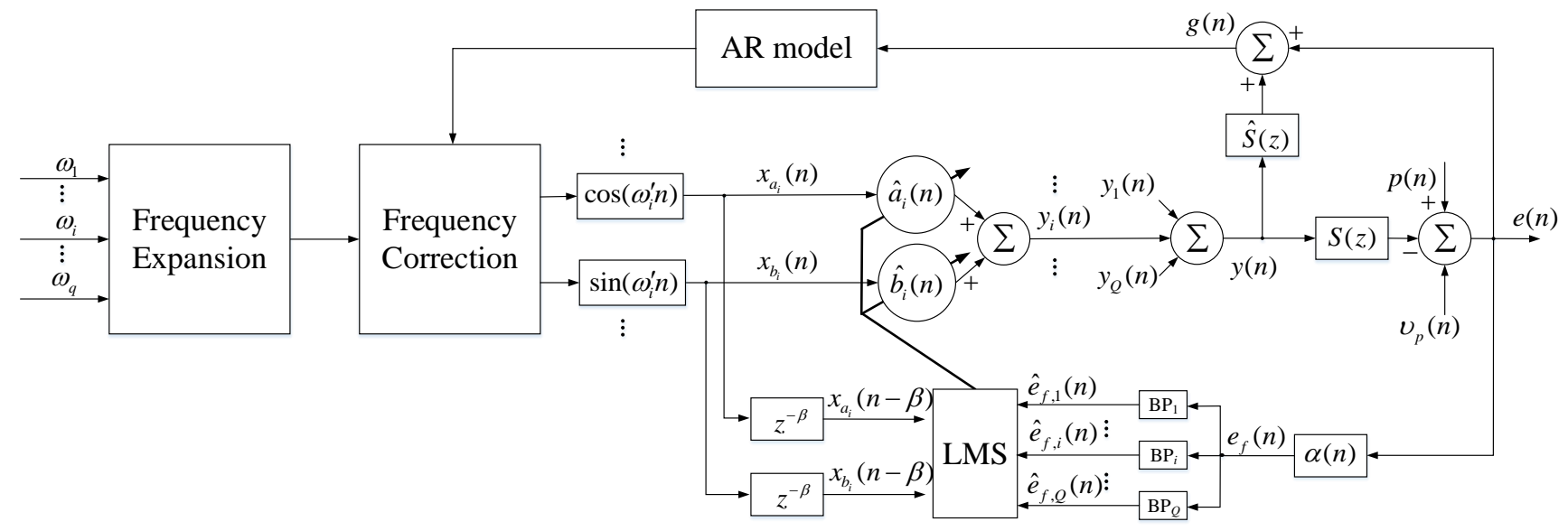

Fig .5 Schematic diagram of proposed NNANC scheme using error separation technique (Proposed-2)

\subsection{Computational Complexity}

Provided that the number of frequency components of primary noise is $Q$, the number of expanded frequencies is $Q^{\prime}$, each frequency need to be searched $D$ times, the length of AR model is $L, \hat{M}$ acts as the order of secondary path estimate, the computational complexity per iteration of the Proposed-1 is computed as follows.

\section{For calculating the output $y(n)$}

Number of multiplications : $2 Q$

Number of additions : $Q$

\section{For calculating the spectrum}

Number of multiplications : $4 L+1$

Number of additions : $L+2$

\section{For maxima determination}

Number of multiplications : $4 Q^{\prime} D$

Number of additions : $3 Q^{\prime} D$

\section{For updating coefficients}

Number of multiplications : $2 Q+\hat{M}+1$

Number of additions : $2 Q+\hat{M}+1$

Number of $\sin (\cdot) / \cos (\cdot): 2 Q$

\section{Total computational load}


Total number of multiplications : $4 D Q^{\prime}+4 Q+4 L+\hat{M}+2$

Total number of additions : $3 D Q^{\prime}+3 Q+L+\hat{M}+3$

Total number of $\sin (\cdot) / \cos (\cdot): 2 Q$

In Proposed-2, the computation load of updating coefficients is estimated as follows:

Number of multiplications : $10 Q+\hat{M}+1$

Number of additions : $8 Q+\hat{M}+1$

Number of $\sin (\cdot) / \cos (\cdot): 2 Q$

The total number of multiplications and additions of Proposed-1 are given by : $4 D Q^{\prime}+12 Q+4 L+\hat{M}+2$ and $3 D Q^{\prime}+9 Q+L+\hat{M}+3$ respectively

Table 1

Comparison of computational complexity one iteration: (a) FSLMS algorithm, (b) second-order VFXLMS algorithm, (c) RFFLNN algorithm, (d) Proposed-1, (e)

Proposed-2

\begin{tabular}{|c|c|c|c|c|c|}
\hline Scheme & FSLMS & VFXLMS & RFFLNN & Proposed-1 & Proposed-2 \\
\hline Multiplications & $N(2 P+1)(\hat{M}+2)+1$ & $\frac{(N+3) N}{2}(\hat{M}+2)+1$ & $\begin{array}{l}N_{1}(2 P+1)(\hat{M}+3) \\
-\hat{M}+\hat{M} N_{2}+3 N_{2}\end{array}$ & $\begin{array}{l}4 D Q^{\prime}+4 Q+4 L \\
+\hat{M}+2\end{array}$ & $\begin{array}{l}4 D Q^{\prime}+12 Q+4 L \\
+\hat{M}+2\end{array}$ \\
\hline Additions & $N(2 P+1)(\hat{M}+1)-1$ & $\frac{(N+3) N}{2}(\hat{M}+1)-1$ & $\begin{array}{l}N_{1}(2 P+1)(\hat{M}+1) \\
+3 N_{2}+1\end{array}$ & $\begin{array}{l}3 D Q^{\prime}+3 Q+L \\
+\hat{M}+3\end{array}$ & $\begin{array}{l}3 D Q^{\prime}+9 Q+L \\
+\hat{M}+3\end{array}$ \\
\hline $\sin (\cdot) / \cos (\cdot)$ & $2 N P$ & 0 & $2 N_{1} P$ & $2 Q$ & $2 Q$ \\
\hline
\end{tabular}

The comparison of computational load of the feedforward structure FSLMS and second-order VFXLMS algorithms, the combination of feedforward structure and feedback structure RFFLNN algorithm [23] and proposed schemes for one iteration is listed in Table 1, where $P$ is the order of FSLMS and RFFLNN algorithms, $N$ and $N_{1}$ are the memory size of reference signal, $N_{2}$ is the memory size of feedback information. It can be noted that the computational load of proposed schemes are immune to memory size. Besides, Proposed-2 is always more than Proposed-1 $8 Q$ multiplications and $6 Q$ additions. Considered $\tau_{i}<0.02 \omega_{e, i}$ and search number $D \leq 5$. Other parameters are set at $Q^{\prime}=15, Q=9, L=30, N=40, N_{1}=35, N_{2}=20$, the number of multiplications and additions of one iteration required in first-order FSLMS algorithm, second-order VFXLMS algorithm, first-order RFFLNN algorithm and proposed schemes separately computed using Table 1 are plotted in Fig. 6 as a function of $\hat{M}$. It is clearly evident that the proposed methods are more computationally efficient than the other three algorithms. 


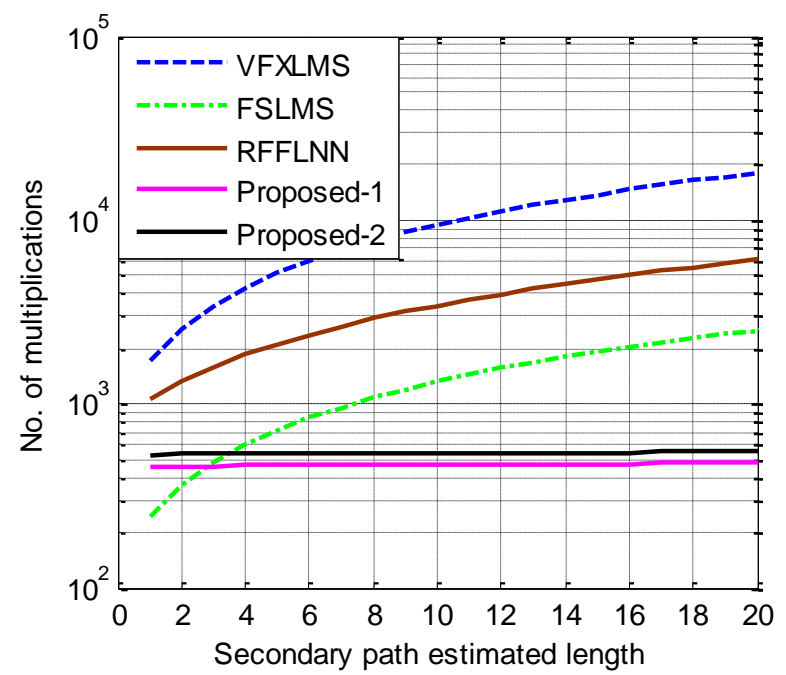

(a) Multiplications

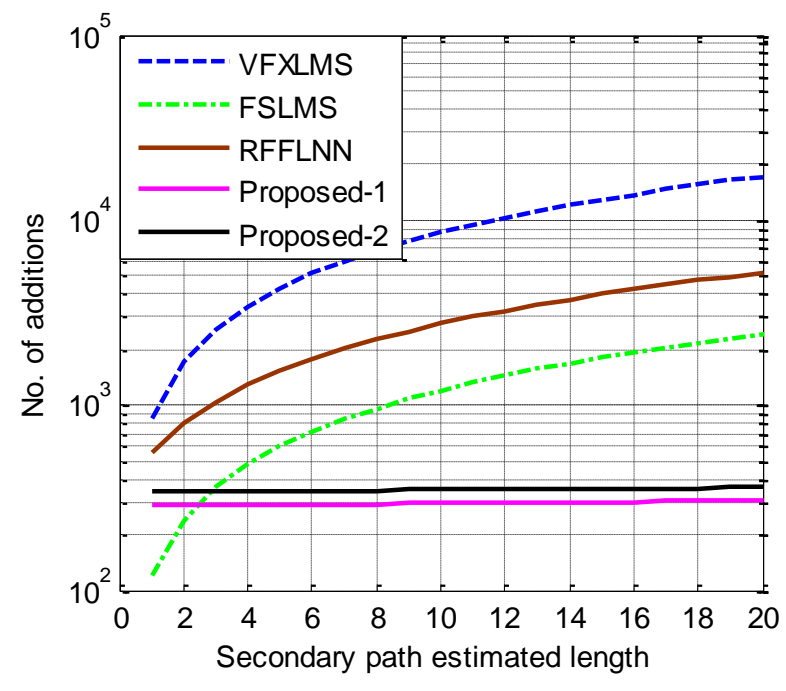

(b) Additions

Fig. 6 Comparison of computational load per iteration

\section{Simulation studies}

In order to analyse the performance of the proposed schemes composed of feedforward and feedback structures, several simulations compared with the NNANC schemes using VFXLMS algorithm, FSLMS algorithm and RFFLNN algorithm are presented in this section. For performance comparison, the normalized mean-square error (NMSE) defined as $\mathrm{NMSE}=10 \log _{10}\left\{E\left[e^{2}(n)\right] / \sigma_{p}^{2}\right\}$ is used, where $\sigma_{p}^{2}$ is the variance of the primary noise at the cancellation point. In each simulation, ten independent trials are conducted to evaluate the NMSE of all the schemes involved in this section. In all examples, the sampling frequency is $f_{s}=2000 \mathrm{~Hz}$ and the background noise $v_{p}(n)$ is a white Gauss noise between -0.1 to 0.1 .

The step sizes of FSLMS algorithm, VFLMS algorithm and RFFLNN algorithm are all set as $\mu_{F}=\mu_{V}=\mu_{R}=0.0009$, and the step sizes of Proposed-1 and Proposed-2 are $\mu_{P}=0.006$.

In all simulations, FSLMS algorithm is first-order, VFXLMS algorithm is second-order and RFFLNN algorithm is first-order, and the secondary path is an FIR filter with non-minimum phase used in paper [28-30].

$$
S(z)=z^{-2}+1.5 z^{-3}-z^{-4}
$$

The secondary path is estimated offline with a white noise whose variance is one, the order $\hat{M}=15$. Forty independent trials are conducted to calculate the mean behaviours of them.

\subsection{Example 1}

In this example, the frequency components of reference signal are $\omega_{1}=0.1 \pi(100 \mathrm{~Hz}), \omega_{2}=0.2 \pi(200 \mathrm{~Hz})$, $\omega_{3}=0.3 \pi(300 \mathrm{~Hz})(q=3)$, whose DFCs are $a_{1}=2.0, b_{1}=-1.0, a_{2}=1.0, b_{2}=-0.5$ and $a_{3}=0.5, b_{3}=0.1$. The transfer 
function of the primary path used in paper [30] is given by

$$
p(n)=x(n-3)-0.3 x(n-4)+0.2 x(n-5)+0.8 x^{2}(n-5)
$$

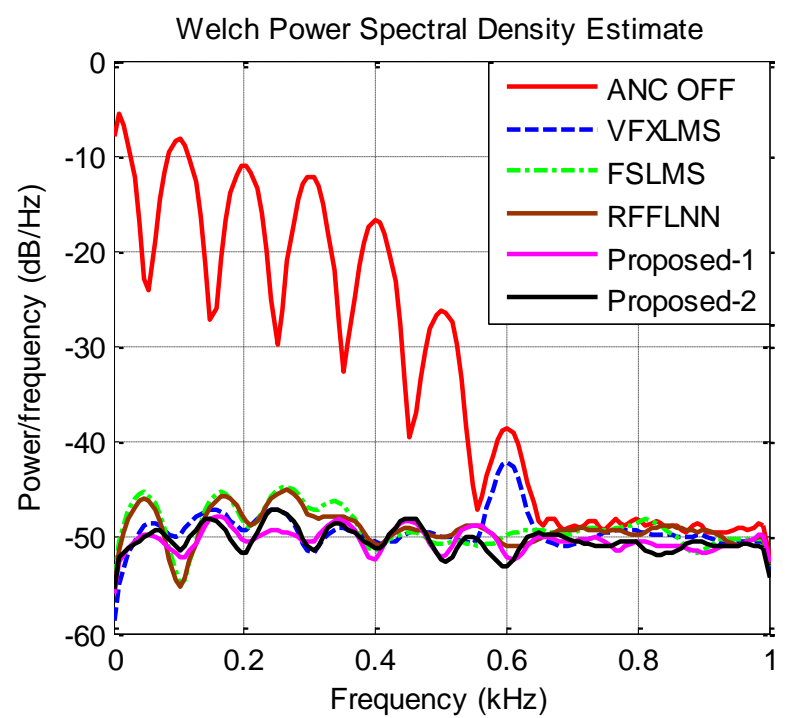

(a) PSD

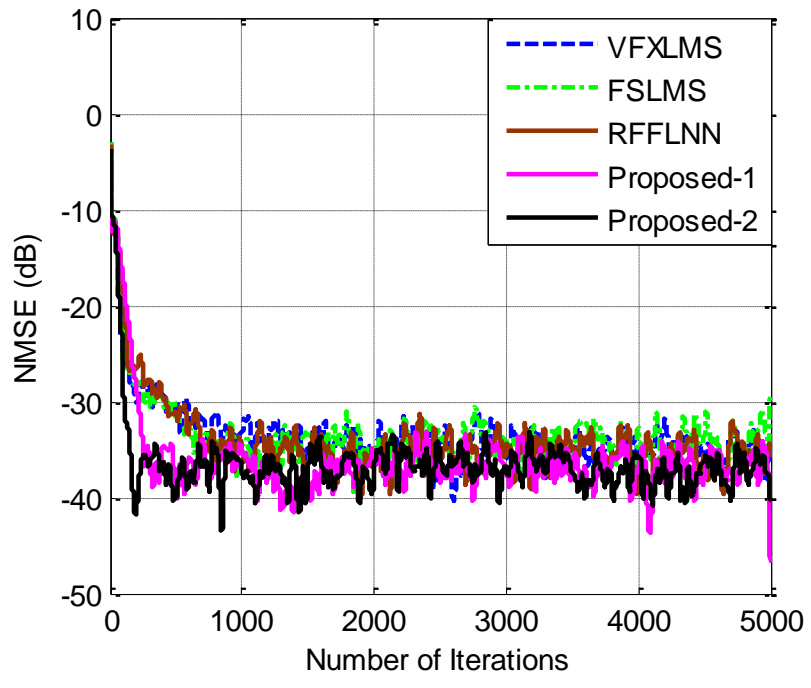

(b) NMSE learning curves

Fig. 7 Performance comparison using second-order nonlinearity primary path and multi-frequency reference signal

Table 2

Frequency components analysis of primary noise $(q=3, T=2)$

\begin{tabular}{|c|c|}
\hline Frequencies & Coefficients and polynomials \\
\hline $100 \mathrm{~Hz}$ & $\begin{array}{c}\left\{d_{1,1}=1, d_{1,2}=0, d_{1,3}=0\right\}, x(n-3), 0.3 x(n-4), 0.2 x(n-5) \\
\left\{d_{2,1}=-1, d_{2,2}=1, d_{2,3}=0\right\}, 0.8 x^{2}(n-5) \\
\left\{d_{3,1}=0, d_{3,2}=-1, d_{3,3}=1\right\}, 0.8 x^{2}(n-5)\end{array}$ \\
\hline $200 \mathrm{~Hz}$ & $\begin{array}{c}\left\{d_{4,1}=0, d_{4,2}=1, d_{4,3}=0\right\}, x(n-3), 0.3 x(n-4), 0.2 x(n-5) \\
\left\{d_{5,1}=2, d_{5,2}=0, d_{5,3}=0\right\}, 0.8 x^{2}(n-5) \\
\left\{d_{6,1}=-1, d_{6,2}=0, d_{6,3}=1\right\}, 0.8 x^{2}(n-5)\end{array}$ \\
\hline $300 \mathrm{~Hz}$ & $\begin{array}{c}\left\{d_{7,1}=1, d_{7,2}=1, d_{7,3}=0\right\}, 0.8 x^{2}(n-5) \\
\left\{d_{8,1}=0, d_{8,2}=0, d_{8,3}=1\right\}, x(n-3), 0.3 x(n-4), 0.2 x(n-5)\end{array}$ \\
\hline $400 \mathrm{~Hz}$ & $\begin{array}{l}\left\{d_{9,1}=0, d_{9,2}=2, d_{9,3}=0\right\}, 0.8 x^{2}(n-5) \\
\left\{d_{10,1}=1, d_{10,2}=0, d_{10,3}=1\right\}, 0.8 x^{2}(n-5)\end{array}$ \\
\hline $500 \mathrm{~Hz}$ & $\left\{d_{11,1}=0, d_{11,2}=1, d_{11,3}=1\right\}, 0.8 x^{2}(n-5)$ \\
\hline $600 \mathrm{~Hz}$ & $\left\{d_{12,1}=0, d_{12,2}=0, d_{12,3}=2\right\}, 0.8 x^{2}(n-5)$ \\
\hline
\end{tabular}


In this simulation, the memory size of FSLMS algorithm and VFLMS algorithm are $N=30$, and that of RFFLNN algorithm is $N_{1}=25, N_{2}=15$. For Proposed-1and Proposed-2, the length of AR model $L=20$ is chosen. The power spectral density (PSD) of the primary noise is shown in Fig. 7 (a), where frequency components contain $100 \mathrm{~Hz}, 200 \mathrm{~Hz}, 300 \mathrm{~Hz}, 400 \mathrm{~Hz}, 500 \mathrm{~Hz}$ and $600 \mathrm{~Hz}$. These frequencies calculated by Eq. (7) is listed in Table 2. The simulated NMSE results of all schemes are presented in Fig. 7 (b). Compared these algorithms, the NMSE obtained by proposed schemes are best at about $-37.0 \mathrm{~dB}$, and that of others at about -35.0dB. In addition, Proposed-1 and Proposed-2 hold faster convergence rate and less computational load.

\subsection{Example 2}

In this example, the frequency of reference signal is $\omega=0.1 \pi(100 \mathrm{~Hz})(q=1)$, whose DFCs are $a=2.0, b=-1.0$. The transfer function of primary path exhibits high order nonlinear, which is modelled by Volterra series

$$
\begin{aligned}
p(n)= & x(n)+0.8 x(n-1)+0.3 x(n-2)+0.4 x(n-3)-0.8 x(n) x(n-1) \\
& +0.9 x(n) x(n-2)+0.7 x(n) x(n-2) x(n-3)
\end{aligned}
$$

The simulation results of PSD and NMSE are shown in Fig. 8 (a) and Fig. 8 (b), where the frequencies of primary noise are only the harmonic of reference signal. In this case, the memory size of FSLMS algorithm, VFXLMS algorithm and RFFLNN algorithm, and the AR model order of Propose- 1 and Proposed-2 are all the same as example 1 . It is noted that the proposed schemes perform better on convergence rate and steady-state error. VFXLMS algorithm performs worst among them at -15.7 dB, because the VFXLMS algorithm is second order and can't generate the third harmonic frequencies. The NMSE of FSLMS algorithm and RFFLNN algorithm are similar at about $-41.0 \mathrm{~dB}$, but that of Prposed-1 and Proposed -2 are $-42.8 \mathrm{~dB}$ and $-42.9 \mathrm{~dB}$ respectively.

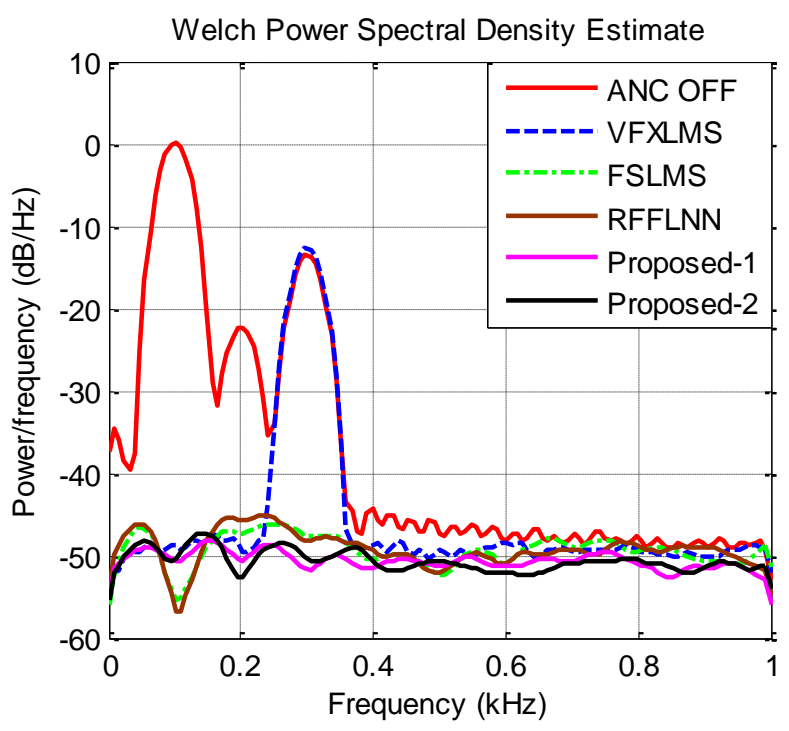

(a) PSD

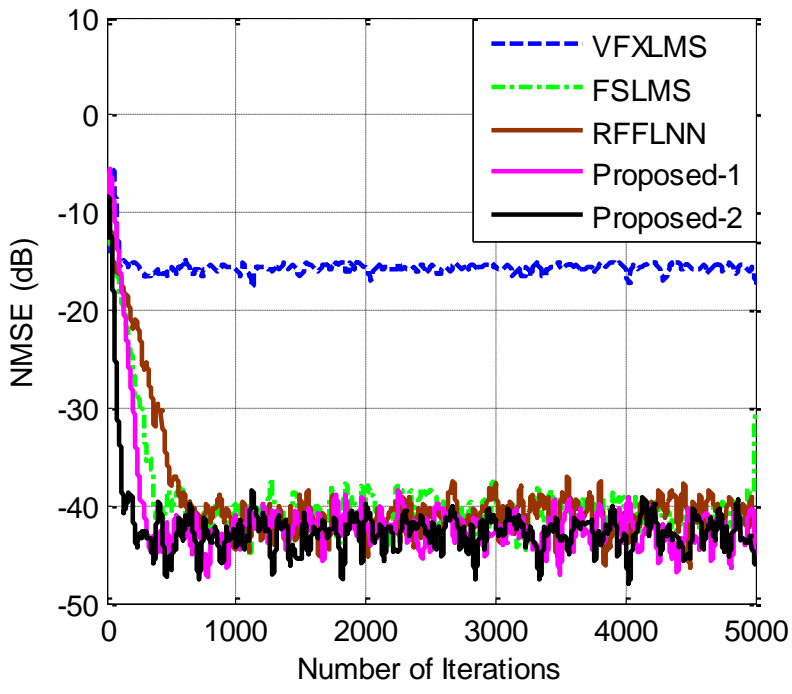

(b) NMSE learning curves

Fig. 8 Performance comparison using high order nonlinearity primary path and single frequency reference signal 


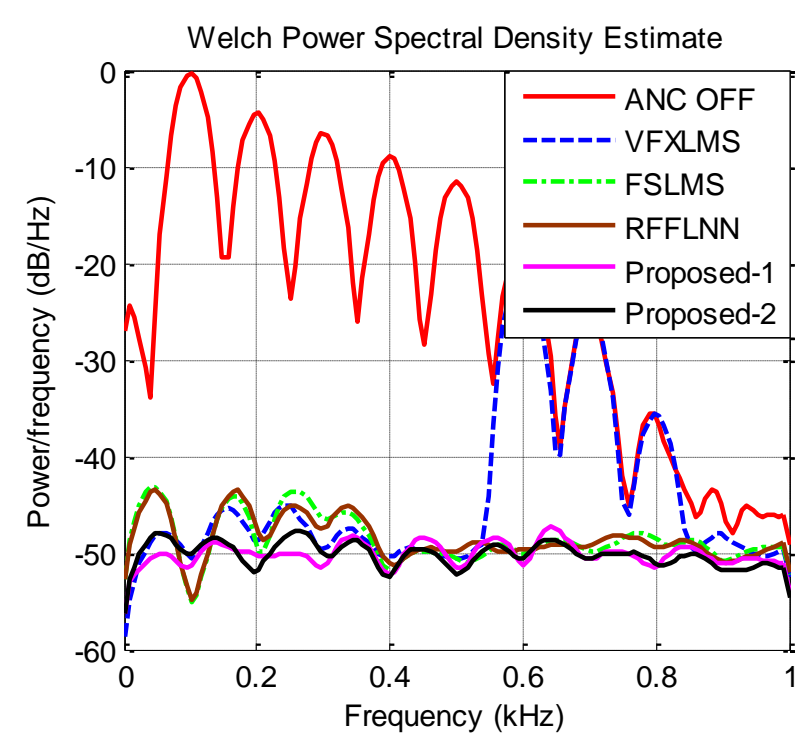

(a) PSD

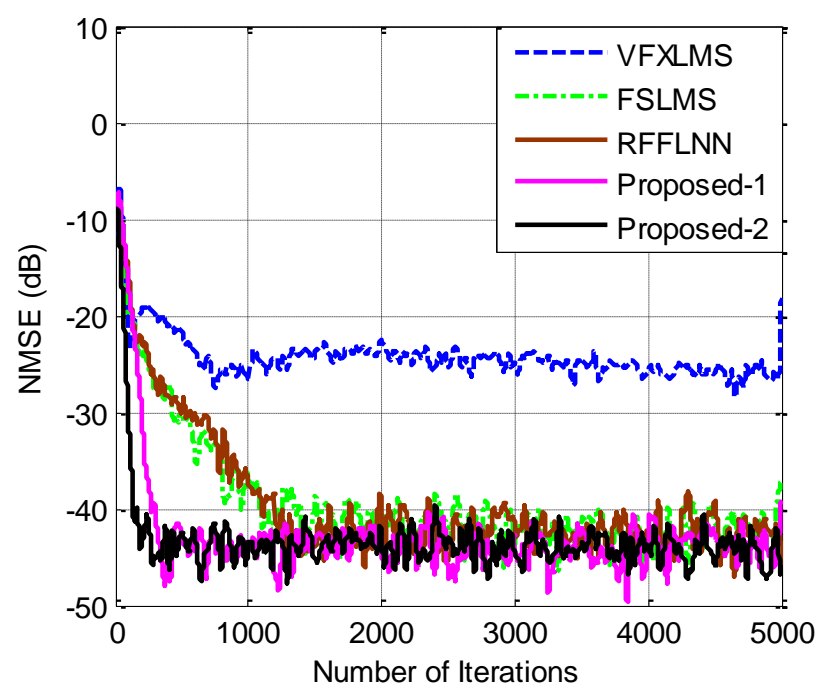

(b) NMSE learning curves

Fig. 9 Performance comparison using high order nonlinearity primary path and multi-frequency reference signal

In this example, the reference signal is the same as example 1 and the primary path is also shown in Eq. (41). The memory size of FSLMS and VFXLMS algorithms is $N=40$, the memory size of RFFLNN algorithm are $N_{1}=35, N_{2}=20$, the order of AR model is $L=30$. PSD and NMSE of this case are shown in Fig. 9 (a) and Fig. 9 (b). The primary noise contains nine frequency components $100 \mathrm{~Hz}, 200 \mathrm{~Hz}, 300 \mathrm{~Hz}, 400 \mathrm{~Hz}, 500 \mathrm{~Hz}, 600 \mathrm{~Hz}, 700 \mathrm{~Hz}, 800 \mathrm{~Hz}$ and $900 \mathrm{~Hz}$, which can be obtained by the similar method introduced in Table 2. It has been observed that, in the case of high order nonlinearity and multi-frequency reference signal, the Proposed-1 and Proposed-2 perform best on convergence rate and NMSE at $-43.6 \mathrm{~dB}$ and $-44.3 \mathrm{~dB}$ respectively. The NMSE obtained by FSLMS algorithm and RFFLNN algorithm are analogous at about $-42.0 \mathrm{~dB}$, but that of VFXLMS algorithm is only at $-25.9 \mathrm{~dB}$ because of lacking of cubic polynomial.

\subsection{Example 4}

In real practical situation, maybe the magnitude of reference signal changes abruptly, this case leads to a non-stationary phenomenon in NNANC system. It is expected that the scheme should be able to be adjusted promptly to track such change. The frequency components of reference signal used in this example are the same as example 1, and the DFCs of each frequency are taken identically to example 1 till 5000 iterations, and then these DFCs change instantly to their opposite number, namely, $a_{1}=-2.0, b_{1}=1.0, a_{2}=-1.0, b_{2}=0.5$ and $a_{3}=-0.5, b_{3}=-0.1$. The primary noise at the cancellation point is generated according to the third-order polynomial model

$$
p(n)=u(n-2)+0.08 u^{2}(n-2)-0.04 u^{3}(n-2)
$$

where $u(n)$ is obtained from the convolution of reference signal $x(n)$ with $f(n)$, and $f(n)$ is the impulse response of the 
transfer function

$$
F(z)=z^{-3}-0.3 z^{-4}+0.2 z^{-5}
$$

The memory size of FSLMS, VFXLMS and RFFLNN algorithms and the AR model order are the same as example 3, The NMSE results of all schemes are plotted in Fig. 10. Among these schemes, Proposed-1 and Proposed-2 show the best capacity for adaptive tracking.

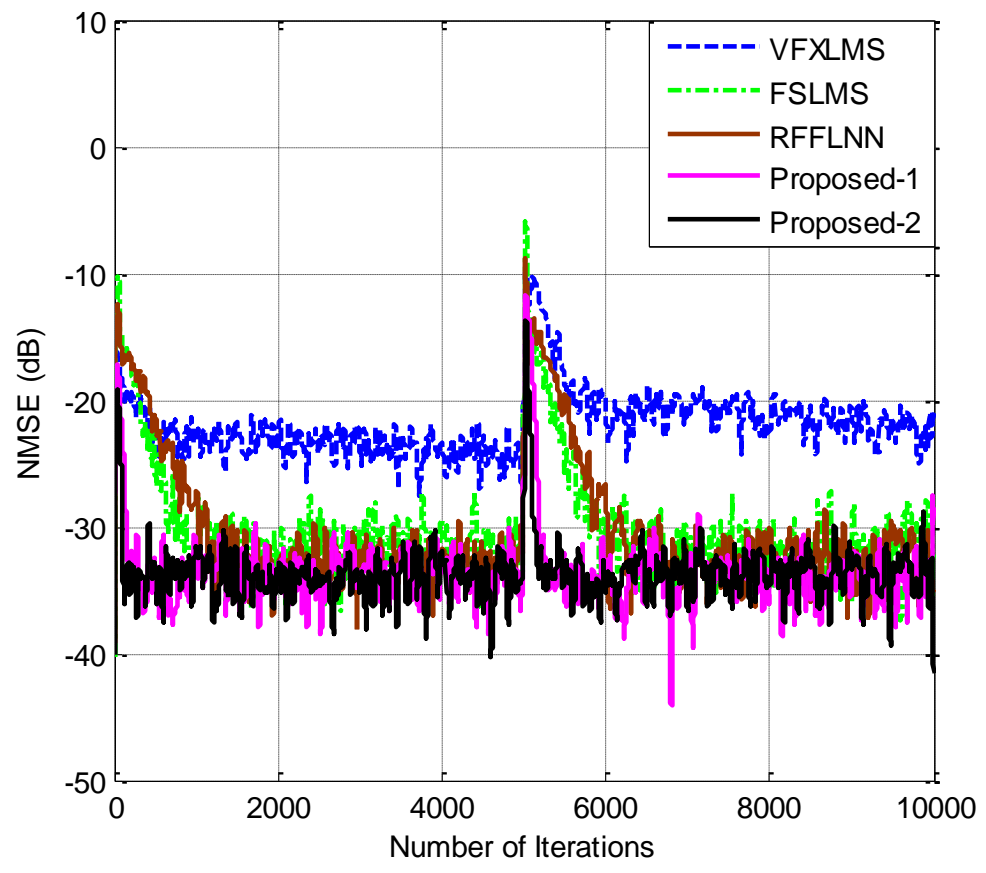

Fig. 10 Performance comparison of NMSE learning curves with non-stationary phenomenon

\section{Conclusion}

In this paper, a novel NNANC scheme composed of feedforward and feedback structures with filtered-error algorithm (Proposed-1) is proposed and its improvement (Proposed-2) using error separation technique composed of bandpass filter banks is also developed. By analysing the computational complexity, it can be clearly noted that Proposed-1 and Proposed-2 have less computational load than other feedforward structure like FSLMS, VFXLMS algorithms and the combination of feedforward structure and feedback structure like RFFLNN algorithm, especially the Proposed-1 which is the least. The results obtained by the simulation studies have shown that proposed schemes outperform others in the case of nonlinear primary path and nonminimum phase secondary path. When VFXLMS algorithm has the same order with the primary path, unfortunately, whose order is often unknown, the performance of VFXLMS algorithm may be comforting, but the computational complexity will increase enormously. FSLMS algorithm, RFFLNN algorithm and proposed schemes are all immune to the order of primary path, but the proposed schemes have better performance on convergence speed and steady-state error. Compared with Proposed-1, the 
computational complexity of Proposed-2 is slightly more, but the performance of narrowband noise cancellation of Proposed-2 is better. Therefore, it is concluded that the Proposed-2 is a suitable candidate with better performance and less computational complexity for NNANC processes.

\section{Acknowledgement}

This work was partially supported by National Science Foundation of PR China (Grant 1171183, 61471140), Aerospace Support Fund (01320214) and Natural Scientific Research Innovation Foundation in Harbin Institute of Technology (HIT.NSRIF201508954).

\section{References}

[1] Kuo, S.M., Morgan, D.R, Active noise control systems: algorithms and DSP implementations, Wiley, New York, 1996.

[2] Kuo, S.M., Morgan, D.R, Active noise control: a tutorial review, Proceedings of IEEE. 87 (6) (1999)

[3] Yegui Xiao, Wang J, A new feedforward hybrid active noise control system, IEEE Signal Processing Letters. 18 (10) (2011) $591-594$

[4] Yegui Xiao, Akira Ikuta, Liying Ma, K. Khorasani, Stochastic analysis of the FXLMS-based narrowband active noise control system, IEEE Transactions on Audio, Speech, and Language Processing.. 16 (5) (2008) 1000-1014.

[5] Yegui Xiao, Liying Ma, Koji Hasegawa, Properties of FXLMS-Based Narrowband Active Noise Control With Online Secondary-Path Modeling, IEEE Transactions on Signal Processing. 57 (8) 2009 2931-2949.

[6] I.T. Ardekani, W.H. Abdulla, Theoretical convergence analysis of FXLMS algorithm, Signal Processing 90 (12) (2010) 3046-3055

[7] Nithin V. George, Ganapati Panda, Advances in active noise control: A survey, with emphasis on recent nonlinear techniques, Signal Processing. 93 (2013) 363-377.

[8] P. Strauch and B. Mulgrew, Nonlinear active noise control in a linear duct, IEEE Transactions on Signal Processing. 46 (9) (1998) 2404-2412.

[9] Snyder SD, Tanaka N, Active control of vibration using a neural network, IEEE Transactions on Neural Networks. 6 (7) (1995) 819-29.

[10] Bouchard M, Pailard B, Dinh CTL, Improved training of neural networks for nonlinear active control of sound and vibration, IEEE Transactions on Neural Networks. 10 (2) (1999) 391-401.

[11] Bouchard M, New recursive-least-squares algorithms for non-linear active control of sound and vibrations using neural networks, IEEE Transactions on Neural Networks. 12 (1) (2001) 135-47.

[12] Chang CY, Neural filtered-U algorithm for the application of active noise control system with correction terms momentum, 
Digital Signal Processing. 20 (10) (2010) 19-26.

[13] D.P. Das, G. Panda, Active mitigation of nonlinear noise processes using a novel filtered-s LMS algorithm, IEEE Transactions on Audio, Speech, and Language Processing. 12 (3) (2004) 313-322.

[14] D.P. Das, G. Panda, D. K. Nayak, Development of frequency domain block filtered-s lms (FBFSLMS) algorithm for active noise control system, IEEE International Conference on Acoustics, Speech and Signal Processing. (2006) 289-292.

[15] D. P. Das, Danielle J. Moreau, Ben S. Cazzolato, Nonlinear active noise control for headrest using virtual microphone control, Control Engineering Practice. 21 (2013) 544-555

[16] D. P. Das, S.R. Mohapatra, A. Routray, T.K. Basu, Filtered-s LMS algorithm for multichannel active control of nonlinear noise processes, IEEE Transactions on Audio, Speech, and Language Processing. 14 (5) (2006) 1875-1880.

[17] G. L. Sicuranza, A. Carini, A generalized FLANN filter for nonlinear active noise control, IEEE Transactions on Audio, Speech, and Language Processing. 19 (8) (2011) 2412-2417.

[18] G. L. Sicuranza, A. Carini, On the BIBO stability condition of adaptive recursive FLANN filters with application to nonlinear active noise control, IEEE Transactions on Audio, Speech, and Language Processing 20 (1) (2012) 234-245.

[19] L. Tan, J. Jiang, Adaptive Volterra filters for active control of nonlinear noise processes, IEEE Transactions on Signal Processing. 49 (8) (2001) 1667-1676

[20] L. Tan, J. Jiang, Filtered-X second-order Volterra adaptive algorithms, Electronics Letters. 33 (8) (1997) 671-672.

[21] Haiquan Zhao, Xiangping Zeng, Xiaoqiang Zhang, Zhengyou He, Tianrui Li, Adaptive extended pipelined second-order Volterra filter for nonlinear active noise controller, IEEE Transactions on Audio, Speech, and Language Processing 20 (4) (2012) 1394-1399

[22] E. P. Reddy, D. P. Das, K. M. M. Prabhu, Fast Adaptive Algorithms for Active Control of Nonlinear Noise Processes, IEEE Transactions on Signal Processing. 56 (9) (2008) 4530-4536.

[23] Haiquan Zhao, Xiangping Zeng, Jiashu Zhang, Adaptive reduced feedback FLNN filter for active control of nonlinear noise processes, Signal Processing. 90 (3) (2010) 834-847

[24] Yegui Xiao, A New Efficient Narrowband Active Noise Control System and its Performance Analysis, IEEE Transactions on Audio, Speech, and Language Processing. 19 (7) (2011) 1865-1874.

[25] D. Zhou, D. Victor, Efficient Adaptive Nonlinear Filters for Nonlinear Active Noise Control, IEEE Transactions on Circuits and Systems. 54 (3) (2007) 669-681.

[26] V.E. Debrunner, D. Zhou, Hybrid filtered error LMS algorithm: another alternative to filtered-x LMS, IEEE Trans. Circuits Syst.-I: Regular Pap. 53 (3) (2006) 653-661.

[27] Haiquan Zhao, Xiangping Zeng, Zhengyou He, Tianrui Li, Adaptive RSOV filter using the FELMS algorithm for nonlinear 
active noise control systems, Mechanical Systems and Signal Processing. 34 (2013) 378-392.

[28] Vinal Patel, Nithin V. George, Nonlinear active noise control using spline adaptive filters, Applied Acoustics. 93 (2015) $38-43$

[29] Nithin V. George, Ganapati Panda, A robust filtered-s LMS algorithm for nonlinear active noise control, Applied Acoustics. 73 (2012) 836-841

[30] Xinghua Zhang, Xuemei Ren, Jing Na, Bo Zhang, Hong Huang, Adaptive nonlinear neuro-controller with an integrated evaluation algorithm for nonlinear active noise systems, Journal of Sound and Vibration. 329 (2010) 5005-5016. 